IRSTI 27.29 .23

\author{
${ }^{1,3}$ M.K. Dauylbayev, ${ }^{2}$ M. Akhmet, ${ }^{4,{ }^{*}}$ A.E. Mirzakulova, ${ }^{1,3}$ A.B. Uaissov \\ ${ }^{1}$ al-Farabi Kazakh National University, Almaty, Kazakhstan \\ ${ }^{2}$ Middle East Technical University, Ankara, Turkey \\ ${ }^{3}$ Institute of Mathematics and Mathematical Modeling, Almaty, Kazakhstan \\ ${ }^{4}$ Abay Kazakh National Pedagogical University, Almaty, Kazakhstan \\ *e-mail: aziza.mirzakulova@mail.ru
}

\title{
Asymptotic convergence of the solution of the initial value problem for singularly perturbed higher-order integro-differential equation
}

\begin{abstract}
The article is devoted to research the Cauchy problem for singularly perturbed higher-order linear integro-differential equation with a small parameters at the highest derivatives, provided that the roots of additional characteristic equation have negative signs. An explicit analytical formula of the solution of singularly perturbed Cauchy problem is obtained. The theorem about asymptotic estimate of a solution of the initial value problem is proved. The nonstandard degenerate initial value problem is constructed. We find the solution of the nonstandard degenerate initial value problem. An estimate difference of the solution of a singularly perturbed and nonstandard degenerate initial value problems is obtained. The asymptotic convergence of solution of a singularly perturbed initial value problem to the solution of the nonstandard degenerate initial value problem is established.

Key words: singular perturbation, small parameter, the initial functions, asymptotics, passage to the limit
\end{abstract}

\section{Introduction}

The differential and integro-differential equations with small parameters at the highest derivatives are used as mathematical models of various problems of physics, astrophysics, chemistry, biology, mechanics, engineering, etc. At present time, these equations are called singularly perturbed.

The initial value problem with initial jumps for a nonlinear ordinary differential equation of the second order with a small parameter was studied by M.I. Vishik and L.A. Lyusternik [1] and K. A. Kassymov [2]. They show that the solution of the original initial value problem tends to the solution of the degenerate equation with changed initial conditions, when the small parameter approaches zero. Such problems became known as the Cauchy problems with initial jumps. The most general cases of the Cauchy problem for singularly perturbed nonlinear systems of ordinary differential and integro-differential equations, as well as for partial differential equations of hyperbolic type were studied by K.A. Kassymov [3-6].

For the first time, boundary value problems with initial jumps for singularly perturbed linear ordinary differential and integro-differential equations of the second order was studied by K. A. Kassymov [7, 8]. A systematic study of boundary value problems with initial jumps Kassymov and his students began in the nineties of the last century. He developed methods for qualitative research and the construction of an asymptotic expansion of solutions of boundary value problems with initial jumps for singularly perturbed ordinary differential equations $[9,10]$. General boundary-value problems for singularly perturbed ordinary differential equations of higher orders are investigated by D. N. Nurgabyl. He singled out a class of singularly perturbed boundary value problems with an initial jump and developed an algorithm for constructing and investigating the asymptotic behavior of solutions of general boundary value problems $[11,12]$. 
K. A. Kassymov and M. K. Dauylbayev for singularly perturbed higher-order integrodifferential equations studied problems of a special type, when the presence of integral terms leads to a qualitative change in the behavior of the solution [13-15]. It is shown that, in the absence of integral terms, the solution of the differential equation obtained in this case grows unboundedly as the small parameter tends to zero and, consequently, has no finite limit. But the solution of the initial integrodifferential problem has a certain limit, but the limit function is not a solution of the usual degenerate equation. The initial jump takes not only a solution, but also integral terms. For singularly perturbed higher-order integro-differential equations, phenomena of initial jumps of any order were first revealed. It is established that this property essentially depends on the order of the derivatives entering under the integral sign.
M. K. Dauylbayev [16, 17] studied boundary value problems with two boundary layers possessing the phenomena of initial jumps. The novelty of these studies is that when the small parameter tends to zero, the fast solution variable grows unlimitedly, not only at one, the so-called initial point, but also at the other end of the considered segment. Thus, a class of singularly perturbed integro-differential equations with initial jump phenomena at both ends of the given segment is singled out. He also developed a method for studying and constructing the asymptotics of the solution of the Cauchy problem with initial jump for singularly perturbed linear differential equations with impulse action [18].

\section{Main results}

Consider the following singularly perturbed the initial value problem:

$$
L_{\varepsilon} y \equiv \sum_{r=1}^{m} \varepsilon^{r} A_{n+r}(t) y^{(n+r)}(t, \varepsilon)+\sum_{k=0}^{n} A_{k}(t) y^{(k)}(t, \varepsilon)=F(t)+\int_{0}^{1} \sum_{j=0}^{n+m-1} H_{j}(t, x) y^{(j)}(x, \varepsilon) d x
$$

$$
y^{(i)}(0, \varepsilon)=\alpha_{i}, i=\overline{0, n+m-1},
$$

where $\varepsilon>0$ is a small parameter, $\boldsymbol{\alpha}_{i}$, $i=\overline{0, n+m-1}$ are known constants, $A_{n+m}(t)=1$.

We will need the following assumptions:

$$
A_{i}(t) \in C^{(n+m-1)}([0,1]), \quad i=\frac{(\mathrm{C} 1)}{0, n+m}, \quad F(t) \in C^{m-1}([0,1])
$$

and $H_{j}(t, x) \in C^{m-1}(D), \quad j=\overline{0, n+m-1}$, where $D=\{0 \leq t \leq 1, \quad 0 \leq x \leq 1\}$.

$$
\text { (C2) } A_{n}(t) \neq 0,0 \leq t \leq 1 \text {. }
$$

(C3) The roots $\mu_{1} \neq \mu_{2} \neq \ldots \neq \mu_{m} \quad$ of "additional characteristic equation $\mu^{m}+A_{n+m-1}(t) \mu^{m-1}+\ldots+A_{n+1}(t) \mu+A_{n}(t)=0$ satisfy the following inequalities $\mu_{1}<0, \quad \mu_{2}<0, \ldots, \quad \mu_{m}<0$.

Similarly the Cauchy problem (1), (2) for ordinary differential equation was considered in [19]. In the particular case, similarly boundary value problem with initial jumps for this case $m=2, \quad l=2 \quad[16,17]$.

We consider the following homogeneous singularly perturbed differential equation associated with (1):

$$
L_{\varepsilon} y(t, \varepsilon) \equiv \sum_{r=1}^{m} \varepsilon^{r} A_{n+r}(t) y^{(n+r)}(t, \varepsilon)+\sum_{k=0}^{n} A_{k}(t) y^{(k)}(t, \varepsilon)=0 .
$$

The fundamental systems of solutions of the equation (3) has the following asymptotic representation as $\varepsilon \rightarrow 0$ : 


$$
\begin{gathered}
y_{i}^{(q)}(t, \varepsilon)=y_{i 0}^{(q)}(t)+O(\varepsilon), \quad i=\overline{1, n}, \quad q=\overline{0, n+m-1} \\
y_{n+r}^{(q)}(t, \varepsilon)=\frac{1}{\varepsilon^{q}} e^{\frac{1}{\varepsilon_{0} j} \mu_{r}(x) d x}\left(\mu_{r}^{q}(t) y_{n+r, 0}(t)+O(\varepsilon)\right), \quad r=\overline{1, m}, \quad q=\overline{0, n+m-1}
\end{gathered}
$$

where $y_{i 0}(t), \quad i=\overline{1, n}$ are solutions of the problems:

$$
\begin{array}{r}
L_{0} y_{i 0}(t)=0, \quad y_{j 0}^{(i-1)}(0)=\delta_{i j}, \\
i=\overline{1, n}, \quad j=\overline{0, n-1,}
\end{array}
$$

$\delta_{i j}$ is a Kronecker symbol, $y_{n+r, 0}(t), \quad r=\overline{1, m}$ are solutions of the following problems:

$$
\begin{gathered}
p_{r}(t) y_{n+r, 0}^{\prime}(t)+q_{r}(t) y_{n+r, 0}(t)=0, \\
y_{n+r, 0}(0)=1, \quad r=\overline{1, m},
\end{gathered}
$$

where

$$
W(t, \varepsilon)=\frac{1}{\varepsilon^{\lambda}} \cdot \bar{W}(t) \pi(t) \omega(t) \exp \left(\frac{1}{\varepsilon} \int_{0}^{t} \bar{\mu}(x) d x\right)(1+O(\varepsilon)) \neq 0
$$

where $\bar{W}(t)$ is the Wronskian,

$$
\begin{gathered}
\bar{W}(t)=\left|\begin{array}{ccc}
y_{10}(t) & \ldots & y_{n 0}(t) \\
\ldots & \ldots & \ldots \\
y_{10}^{(n-1)}(t) & \ldots & y_{n 0}^{(n-1)}(t)
\end{array}\right|, \quad \lambda=\frac{2 n+m-1}{2}, \\
\bar{\mu}(x)=\mu_{1}(x)+\ldots+\mu_{m}(x)=\sum_{k=1}^{m} \mu_{k}(x), \\
\pi(t)=\prod_{k=1}^{m} y_{n+k, 0}(t) \mu_{k}^{n}(t),
\end{gathered}
$$

the determinant $\omega(t)$ is the $m$-th order Vandermond determinant,

$$
\omega(t)=\left|\begin{array}{ccc}
1 & \cdots & 1 \\
\mu_{1}(t) & \ldots & \mu_{m}(t) \\
\cdots & \cdots & \cdots \\
\mu_{1}^{m-1}(t) & \cdots & \mu_{m}^{m-1}(t)
\end{array}\right| \neq 0 .
$$

$$
p_{r}(t)=\sum_{i=0}^{m} A_{n+i}(t)(n+i) \mu_{r 0}^{n+i-1}(t), \quad r=\overline{1, m}
$$

$$
q_{r}(t)=\boldsymbol{\mu}_{r 0}^{\prime}(t) \sum_{i=0}^{m} A_{n+i}(t) C_{n+i}^{2} \boldsymbol{\mu}_{r 0}^{n+i-2}+A_{n-1}(t) \boldsymbol{\mu}_{r 0}^{n-1}(t)
$$

$$
r=\overline{1, m}
$$

$$
C_{n+i}^{2}=\frac{(n+i) !}{2 !(n+i-2) !}, \quad i=\overline{0, m}
$$

In view of (4), for the Wronskian $W(t, \varepsilon)$ the following asymptotic representation holds as $\varepsilon \rightarrow 0$ :
Definition. The functions $K_{i}(t, s, \boldsymbol{\varepsilon})$, $i=\overline{1, n+m}$ are called initial functions, if they satisfy the following problem:

$$
\begin{gathered}
L_{\varepsilon} K_{i}(t, s, \varepsilon)=0, \quad i=\overline{1, n+m}, \quad 0 \leq s<t \leq 1 \\
K_{i}^{(j)}(s, s, \varepsilon)=\delta_{i-1, j}, \quad j=\overline{0, n+m-1}
\end{gathered}
$$

and that can be represented in the form:

$$
K_{i}(t, s, \varepsilon)=\frac{W_{i}(t, s, \varepsilon)}{W(s, \varepsilon)}, \quad i=\overline{1, n+m}
$$

$W_{i}(t, s, \varepsilon)$ is the $n+m-$ th order determinant obtained from the Wronskian $W(s, \varepsilon)$ by replacing the $i$-th row with $y_{1}(t, \boldsymbol{\varepsilon}), \quad y_{2}(t, \boldsymbol{\varepsilon})$, $\ldots, y_{n+m}(t, \boldsymbol{\varepsilon})$.

In view of (6), (7), for the initial functions $K_{i}^{(q)}(t, s, \varepsilon), \quad i=\overline{1, n+m}$ the following asymptotic representation hold as $\varepsilon \rightarrow 0$ : 


$$
\begin{aligned}
& K_{i}^{(q)}(t, s, \boldsymbol{\varepsilon})=\frac{\bar{W}_{i}^{(q)}(t, s)}{\bar{W}(s)}+\varepsilon^{n-q} \sum_{k=1}^{m} e^{\frac{1}{\varepsilon} \int_{s}^{t} \mu_{k}(x) d x} \frac{y_{n+k, 0}(t) \boldsymbol{\mu}_{k}^{q}(t)}{y_{n+k, 0}(s) \boldsymbol{\mu}_{k}^{n}(s)} \cdot \frac{\boldsymbol{\omega}_{1 k}(s)}{\boldsymbol{\omega}(s)} \cdot \frac{\overline{W_{i}}(s)}{\bar{W}(s)}+O\left(\boldsymbol{\varepsilon}+\boldsymbol{\varepsilon}^{n+1-q} \sum_{k=1}^{m} e^{\frac{1}{\varepsilon} \int_{s}^{t} \mu_{k}(x) d x}\right), \\
& i=\overline{1, n}, \quad q=\overline{0, n+m-1} \text {; } \\
& K_{n+r}^{(q)}(t, s, \boldsymbol{\varepsilon})=\boldsymbol{\varepsilon}^{r}\left[(-1)^{r} \frac{\boldsymbol{\omega}_{r+1}(s)}{\boldsymbol{\omega}(s) A_{n}(s)} \cdot \frac{\bar{W}_{n}^{(q)}(t, s)}{\bar{W}(s)}+\boldsymbol{\varepsilon}^{n-1-q} \sum_{k=1}^{m} e^{\frac{1}{\varepsilon_{s}^{t}} \mu_{k}(x) d x}+\frac{y_{n+k, 0}(t) \boldsymbol{\mu}_{k}^{q}(t)}{y_{n+k, 0}(s) \boldsymbol{\mu}_{k}^{n}(s)} \cdot \frac{\boldsymbol{\omega}_{r k}(s)}{\boldsymbol{\omega}(s)}+O\left(\boldsymbol{\varepsilon}+\boldsymbol{\varepsilon}^{n-q} \sum_{k=1}^{m} e^{\frac{1}{\varepsilon} \int_{s}^{t} \mu_{k}(x) d x}\right)\right], \\
& r=\overline{1, m}, q=\overline{0, n+m-1} \\
& y(t, \boldsymbol{\varepsilon})=\sum_{i=1}^{n+m} C_{i} K_{i}(t, 0, \boldsymbol{\varepsilon})+ \\
& +\frac{1}{\boldsymbol{\varepsilon}^{m}} \int_{0}^{t} \mathbf{K}_{n+m}(t, s, \boldsymbol{\varepsilon}) z(s, \boldsymbol{\varepsilon}) d s
\end{aligned}
$$
determinant obtained from the following determinant

$$
\left|\begin{array}{lll}
1 & \cdots & 1 \\
\mu_{1}(s) & \cdots & \mu_{m}(s) \\
\cdots & \cdots & \cdots \\
\mu_{1}^{m}(s) & \cdots & \mu_{m}^{m}(s)
\end{array}\right|
$$

by deleting $i$-th row, $\bar{W}_{n}^{(q)}(t, s)$ is the determinant obtained from the Wronskian $\bar{W}(s)$ by replacing the $n$-th row with $y_{10}^{(q)}(t), \ldots, y_{n}^{(q)}(t), \bar{W}_{i}(t)$ is the $n+1-$ th order determinant obtained from the following determinant

$$
\left|\begin{array}{cccc}
y_{10}(t) & \ldots & y_{n 0}(t) \\
y_{10}^{\prime}(t) & \ldots & y_{n 0}^{\prime}(t) \\
\ldots & \ldots & & \ldots \\
y_{10}^{(n)}(t) & \ldots & y_{n 0}^{(n)}(t)
\end{array}\right|
$$

by deleting the $i$-th row.

Let us denote by the right-hand side of the equation (1):

$$
z(t, \varepsilon)=F(t)+\int_{0}^{1} \sum_{j=0}^{n+m-1} H_{j}(t, x) y^{(j)}(x, \varepsilon) d x
$$

We seek to find the solution of the differential $L_{\varepsilon} y=z(t, \varepsilon)$ equation in the form: where $K_{i}(t, s, \varepsilon), \quad i=\overline{1, n+m}$ are the initial functions, $z(t, \varepsilon)$ is a unknown function. Substituting (11) into (10), we obtain that $z(t, \varepsilon)$ satisfies the following Fredholm integral equation of the second kind:

$$
z(t, \varepsilon)=f(t, \varepsilon)+\int_{0}^{1} H(t, s, \varepsilon) z(s, \varepsilon) d s
$$

where

$$
f(t, \varepsilon)=F(t)+\sum_{i=1}^{n+m} C_{i} \int_{0}^{1} \sum_{j=0}^{n+m-1} H_{j}(t, x) K_{i}^{(j)}(x, 0, \varepsilon) d x
$$

$$
H(t, s, \varepsilon)=\frac{1}{\varepsilon^{m}} \int_{s}^{1} \sum_{j=0}^{n+m-1} H_{j}(t, x) K_{n+m}^{(j)}(x, s, \varepsilon) d x .
$$

(C4) 1 is not an eigenvalue of the kernel $H(t, s, \varepsilon)$.

In view of condition (C4) integral equation (12) has an unique solution, that can be represented in the form

$$
z(t, \varepsilon)=f(t, \varepsilon)+\int_{0}^{1} R(t, s, \varepsilon) f(s, \varepsilon) d s
$$

where $R(t, s, \varepsilon)$ is a resolvent of the kernel $H(t, s, \varepsilon)$.

Substituting (13) into (11), we obtain the analytical formula of solution: 


$$
y(t, \varepsilon)=\sum_{i=1}^{n+m} C_{i}\left(K_{i}(t, 0, \varepsilon)+\frac{1}{\varepsilon^{m}} \int_{0}^{t} K_{n+m}(t, s, \varepsilon) \bar{\varphi}_{i}(s, \varepsilon) d s\right)+\frac{1}{\varepsilon^{m}} \int_{0}^{t} K_{n+m}(t, s, \varepsilon) \bar{F}(s, \varepsilon) d s
$$

where $C_{i}, \quad i=\overline{1, n+m}$ are unknown constants, $K_{i}(t, s, \varepsilon), \quad i=\overline{1, n+m}$ are the initial functions,

$$
\begin{gathered}
\overline{\boldsymbol{\phi}}_{i}(s, \boldsymbol{\varepsilon})=\int_{0}^{1} \sum_{j=0}^{n+m-1} \bar{H}_{j}(s, x, \boldsymbol{\varepsilon}) K_{i}^{(j)}(x, s, \boldsymbol{\varepsilon}) d x, \\
i=\overline{1, n+m} \\
\bar{H}_{j}(s, x, \boldsymbol{\varepsilon})=H_{j}(s, x)+\int_{0}^{1} R(s, p, \boldsymbol{\varepsilon}) H_{j}(p, x) d p \\
\bar{F}(s, \boldsymbol{\varepsilon})=F(s)+\int_{0}^{1} R(s, p, \boldsymbol{\varepsilon}) F(p) d p .
\end{gathered}
$$

By using initial conditions (2) in (14), we find the constants $C_{i}=\alpha_{i-1}, \quad i=\overline{1, n+m-1}$.

Theorem 1. (Theorem about the analytical formula of solution) Let assumptions (C1)-(C4) hold. Then the Cauchy problem (1), (2) on the segment $0 \leq t \leq 1$ has an unique solution and is expressed by the formula:

$$
\begin{gathered}
y(t, \boldsymbol{\varepsilon})= \\
=\sum_{i=1}^{n+m} \boldsymbol{\alpha}_{i-1}\left(K_{i}(t, 0, \boldsymbol{\varepsilon})+\frac{1}{\boldsymbol{\varepsilon}^{m}} \int_{0}^{t} K_{n+m}(t, s, \boldsymbol{\varepsilon}) \overline{\boldsymbol{\phi}}_{i}(s, \boldsymbol{\varepsilon}) d s\right)+( \\
+\frac{1}{\boldsymbol{\varepsilon}^{m}} \int_{0}^{t} K_{n+m}(t, s, \boldsymbol{\varepsilon}) \bar{F}(s, \boldsymbol{\varepsilon}) d s
\end{gathered}
$$

where $K_{i}(t, s, \varepsilon), \quad i=\overline{1, n+m}$ are the initial functions, functions $\bar{H}_{j}(s, x, \varepsilon), \quad j=\overline{0, n+m-1}$, $\bar{\varphi}_{i}(s, \varepsilon), \bar{F}(s, \varepsilon)$ is defined by the formula (15).

Theorem 2. (Theorem about asymptotic estimations of solution) Let assumptions (C1)(C4) hold. Then the Cauchy problem (1), (2) and its derivatives the following asymptotic estimation hold as $\varepsilon \rightarrow 0$ :

$$
\begin{gathered}
\left|y^{(q)}(t, \boldsymbol{\varepsilon})\right| \leq C\left(\sum_{i=0}^{n-1}\left|\boldsymbol{\alpha}_{i}\right|+\sum_{r=1}^{m} \boldsymbol{\varepsilon}^{r} \cdot\left|\boldsymbol{\alpha}_{n-1+r}\right|+\max _{0 \leq \leq \leq 1}|F(t)|\right)+C \boldsymbol{\varepsilon}^{n-q} e^{-\gamma \frac{t}{\varepsilon}}\left(\sum_{i=0}^{n}\left|\boldsymbol{\alpha}_{i}\right|+\sum_{r=1}^{m-1} \boldsymbol{\varepsilon}^{r} \cdot\left|\boldsymbol{\alpha}_{n+r}\right|+\max _{0 \leq \leq \leq 1}|F(t)|\right) \cdot\left|\sum_{k=1}^{m} \frac{\overline{\boldsymbol{\mu}}_{k}^{q}(t) \boldsymbol{\omega}_{m k}(t)}{\overline{\boldsymbol{\mu}}_{k}^{n+1}(0)}\right|, \\
q=\overline{0, n+m-1}
\end{gathered}
$$

where $C>0, \gamma>0$ are constant independent of $\varepsilon$, $\left.\sum_{k=1}^{m} \frac{\bar{\mu}_{k}^{q}(t) \omega_{m k}(t)}{\bar{\mu}_{k}^{n+1}(0)}\right|_{t=0} \equiv 0, \quad q=\overline{n+1, n+m-1}$.

Proof: In view of (7)-(9) and conditions (C1)(C3), for the initial functions $K_{i}(t, s, \varepsilon), \quad i=\overline{1, n+m}$ the following asymptotic estimation hold:

$$
\begin{aligned}
\left|K_{i}^{(q)}(t, s, \boldsymbol{\varepsilon})\right| & \leq C\left(1+\varepsilon^{n-q} \exp \left(-\frac{t-s}{\varepsilon}\right)\right), \\
i & =\overline{1, n}, q=\overline{0, n+m-1}, \\
\left|K_{n+r}^{(q)}(t, s, \boldsymbol{\varepsilon})\right| & \leq C \boldsymbol{\varepsilon}^{r}\left(1+\varepsilon^{n-1-q} \exp \left(-\frac{t-s}{\varepsilon}\right)\right), \\
r & =\overline{1, m}, \quad q=\overline{0, n+m-1} .
\end{aligned}
$$

By applying the asymptotic estimations of the initial functions (18), (19) in (15), we obtain the following asymptotic estimations for the functions $\bar{\varphi}_{i}(s, \varepsilon), \quad i=\overline{1, n+m}$ :

$$
\begin{aligned}
\left|\bar{\varphi}_{i}(s, \varepsilon)\right| & \leq C, \quad i=\overline{1, n}, \\
\left|\bar{\varphi}_{n+r}(s, \varepsilon)\right| & \leq C \varepsilon^{r}, \quad r=\overline{1, m}
\end{aligned}
$$

By applying (18)-(20) in (16), we obtain asymptotic estimations of the solution (17). Theorem 2 is proved.

We consider the following nonstandard unperturbed initial value problem:

$$
\begin{gathered}
L_{0}[\bar{y}(t)] \equiv \sum_{k=0}^{n} A_{k}(t) \bar{y}^{-(k)}(t)= \\
=F(t)+\int_{0}^{1} \sum_{j=0}^{n+m-1} H_{j}(t, x) \bar{y}^{-(j)}(x) d x,
\end{gathered}
$$




$$
\bar{y}^{(i)}(0)=\alpha_{i}, \quad i=\overline{0, n-1}
$$

We introduce the initial functions for the problem (21), (22), similar to functions (7):

$$
\bar{K}_{i}(t, s)=\frac{\overline{W_{i}}(t, s)}{\bar{W}(s)}, \quad i=\overline{1, n}
$$

where $\bar{W}_{i}(t, s)$ is the $n$-th order determinant obtained from the Wronskian $\bar{W}(s)$ by replacing the $i$-th row with $y_{10}(t), \quad y_{20}(t), \ldots, \quad y_{n 0}(t)$.
It is clear that the initial $\bar{K}_{i}(t, s), \quad i=\overline{1, n}$ functions will be the solution for the following problem:

$$
\begin{gathered}
L_{0} \bar{K}_{i}(t, s)=0, \quad i=\overline{1, n}, t \neq s \\
\bar{K}_{i}^{(j-1)}(s, s)=\delta_{i, j-1}, \quad i=\overline{1, n}, \quad j=\overline{1, n} .
\end{gathered}
$$

Differentiating $m-1$ times the equation (21) with respect to variable $t$, then backwards integrating $m-1$ times on the segment $[0, t]$, we get the auxiliary equation:

$$
L_{0}[\bar{y}(t)]=F(t)+\sum_{k=0}^{m-2} B_{k} \frac{t^{k}}{k !}+\int_{0}^{t} \frac{(t-s)^{m-2}}{(m-2) !} Q[\bar{y}(s)] d s
$$

where $B_{k}, k=\overline{0, m-2}$ are arbitrary constants, operator $Q$ is a linear integro-differential operator

$$
Q[\bar{y}(t)]=\int_{0}^{1} \sum_{j=0}^{n+m-1} H_{j}^{(m-1)}(t, x) \bar{y}^{(j)}(x) d x
$$

symbol $H_{j}^{(m-1)}(t, x)$ means the $m-1-$ th order derivative with respect to variable $t$ of the function $H_{j}(t, x)$.
If the constants $B_{k}, \quad k=\overline{0, m-2}$ satisfy the conditions

$$
B_{k}=\int_{0}^{1} \sum_{j=0}^{n+m-1} H_{j}^{(k)}(0, x) \bar{y}^{(j)}(x) d x, \quad k=\overline{0, m-2}
$$

then the equations (21) and (24) will be equivalent. Let us consider the equation (24) with initial conditions (22).

The solution of the Cauchy problem (22), (24) is as follows:

$$
\bar{y}(t)=\sum_{i=1}^{n} C_{i} \bar{K}_{i}(t, 0)+\varphi(t)+\sum_{k=0}^{m-2} B_{k} \varphi_{k}(t)+\int_{0}^{t} \frac{\hat{K}_{n}(t, s)}{A_{n}(t)} Q[\bar{y}(s)] d s
$$

where $\bar{K}_{i}(t, s), \quad i=\overline{1, n}$ are the initial functions, operator $Q$ is defined by the formula (25),

$$
\begin{gathered}
\phi(t)=\int_{0}^{t} \frac{\bar{K}_{n}(t, s)}{A_{n}(t)} F(s) d s, \\
\phi_{k}(t)=\int_{0}^{t} \frac{\bar{K}_{n}(t, s)}{A_{n}(t)} \cdot \frac{s^{k}}{k !} d s, \quad k=\overline{0, m-2} \\
\hat{K}_{n}(t, s)=\int_{s}^{t} \bar{K}_{n}(t, p) \frac{(p-s)^{m-2}}{(m-2) !} d p .
\end{gathered}
$$

Function $\hat{K}_{n}(t, s)$ satisfy the following problem:

$$
\begin{gathered}
L_{0}[\bar{y}(t)]=\frac{(t-s)^{m-2}}{(m-2) !}, \\
\hat{K}_{n}^{(j)}(s, s)= \begin{cases}0, & j=\overline{0, m-2} \\
1, & j=m-1\end{cases}
\end{gathered}
$$

Substituting (27) into (22), in view of the condition of the initial functions $\bar{K}_{i}(t, s), \quad i=\overline{1, n}$, we find the constants $C_{i}=\alpha_{i-1}, \quad i=\overline{1, n}$. We put defined constants $C_{i}=\alpha_{i-1}, \quad i=\overline{1, n}$ in (27): 


$$
\bar{y}(t)=\sum_{i=1}^{n} \alpha_{i-1} \bar{K}_{i}(t, 0)+\varphi(t)+\sum_{k=0}^{m-2} B_{k} \varphi_{k}(t)+\int_{0}^{t} \frac{\hat{K}_{n}(t, s)}{A_{n}(t)} Q[\bar{y}(s)] d s
$$

where $\bar{K}_{i}(t, s), \quad i=\overline{1, n}$ are the initial functions, Applying operator $Q$ to the equation (29), we arrive operator $Q$ is defined by the formula (25), functions Fredholm integral equation of the second type $\varphi(t), \quad \varphi_{k}(t), \quad k=\overline{0, m-2}, \quad \hat{K}_{n}(t, s) \quad$ are defined by the formula (28).

$$
u(t)=\sum_{i=1}^{n} \alpha_{i-1} \widetilde{K}_{i}(t, 0)+\omega(t)+\sum_{k=0}^{m-2} B_{k} \omega_{k}(t)+\int_{0}^{1} M(t, s) u(s) d s
$$

where

$$
M(t, s)=\left\{\begin{array}{l}
\int_{s}^{0} \sum_{j=0}^{n+m-1} H_{j}^{(m-1)}(t, \tau) \hat{K}_{n}^{(j)}(\tau, s) d \tau+H_{n+m-1}(t, s), \quad s \leq 0 \\
\int_{s}^{1} \sum_{j=0}^{n+m-1} H_{j}^{(m-1)}(t, \tau) \hat{K}_{n}^{(j)}(\tau, s) d \tau+H_{n+m-1}(t, s), \quad s \geq 0
\end{array},\right.
$$

functions

$u(t), \quad \boldsymbol{\omega}(t), \quad \boldsymbol{\omega}_{k}(t), \quad k=\overline{0, m-2}, \quad \tilde{K}_{i}(t, 0), \quad i=\overline{1, n} \quad$ are expressed the following form:

$$
\begin{aligned}
& u(t)=Q[\bar{y}(t)], \quad \omega(t)=Q[\varphi(t)], \\
& \omega_{k}(t)=Q\left[\varphi_{k}(t)\right], \quad k=\overline{0, m-2}, \\
& \widetilde{K}_{i}(t, 0)=Q\left[\bar{K}_{i}(t, 0)\right], \quad i=\overline{1, n} .
\end{aligned}
$$

(C5) 1 is not an eigenvalue of the kernel $M(t, s)$ We introduce the operator:

$$
\begin{aligned}
& A v(t) \equiv v(t)+\int_{0}^{1} \bar{R}(t, s) v(s) d s, \quad \text { (33) } \quad \begin{array}{l}
\text { obtain the solution of the unperturbed problem (21), } \\
\bar{y}(t)=\sum_{i=1}^{n} \alpha_{i-1} \breve{K}_{i}(t, 0)+\varphi(t)+\int_{0}^{t} \frac{\hat{K}_{n}(t, s)}{A_{n}(t)} A \omega(s) d s+\sum_{k=0}^{m-2} B_{k}\left(\varphi_{k}(t)+\int_{0}^{t} \frac{\hat{K}_{n}(t, s)}{A_{n}(t)} A \omega_{k}(s) d s\right)
\end{array}
\end{aligned}
$$

where

$$
\breve{K}_{i}(t, 0)=\bar{K}_{i}(t, 0)+\int_{0}^{t} \frac{\hat{K}_{n}(t, s)}{A_{n}(t)} A \widetilde{K}_{i}(s, 0) d s, \quad i=\overline{1, n} .
$$

We introduce the following symbols: where function $\bar{R}(t, s)$ is a resolvent of the kernel $M(t, s)$. In view of the condition (C5), then the integral equation (30) has an unique solution and that can be represented in the form:

$$
\begin{aligned}
& u(t)=\sum_{i=1}^{n} \alpha_{i-1} A \tilde{K}_{i}(t, 0)+ \\
& +A \omega(t)+\sum_{k=0}^{m-2} B_{k} A \omega_{k}(t)
\end{aligned}
$$

where operator $A$ is defined by the formula (33), $\omega(t), \quad \omega_{k}(t), \quad k=\overline{0, m-2}, \quad \widetilde{K}_{i}(t, 0), \quad i=\overline{1, n} \quad$ are defined by the formula (32).

Substituting the solution (34) into (29), we 
Then the solution (35) is as follows:

$$
\bar{y}(t)=\eta(t)+\sum_{k=0}^{m-2} B_{k} \eta_{k}(t) .
$$

If applying operator $Q$ to the functions $\eta(t), \quad \eta_{k}(t)$ in (36), we get the following equalities:

$$
\begin{gathered}
A \boldsymbol{\omega}(t)=Q[\boldsymbol{\eta}(t)], \quad A \boldsymbol{\omega}_{k}(t)=Q\left[\boldsymbol{\eta}_{k}(t)\right], \\
k=\overline{0, m-2}
\end{gathered}
$$

where operator $A$ is defined by the formula (33).

Taking into account formulas (36) and (38), it is easy to verify that the function (37) satisfies equation (29). That this function is a solution of the equation (21), it is necessary to subordinate the constants $B_{k}, \quad k=\overline{0, m-2}$ to the conditions (26). Substituting (37) into (26), we get the following system for determining $B_{k}, \quad k=\overline{0, m-2}$ :

$$
\begin{aligned}
& B_{k}-\sum_{i=0}^{m-2} a_{k i} \cdot B_{i}=a_{k}, \quad k=\overline{0, m-2} \\
& L_{\varepsilon} u \equiv \sum_{r=1}^{m} \varepsilon^{r} A_{n+r}(t) u^{(n+r)}+\sum_{k=0}^{n} A_{k}(t) u^{(k)}=-\sum_{r=1}^{m} \varepsilon^{r} A_{n+r}(t) \bar{y}^{-(n+r)}+\int_{0}^{1} \sum_{j=0}^{n+m-1} H_{j}(t, x) u^{(j)}(x, \varepsilon) d x \\
& u^{(i)}(0, \varepsilon)=0, \quad i=\overline{0, n-1}, \\
& u^{(i)}(0, \varepsilon)=\alpha_{i}-\bar{y}^{(i)}(0), \quad i=\overline{n, n+m-1} . \\
& \left|u^{(q)}(t, \boldsymbol{\varepsilon})\right| \leq C\left(\sum_{r=1}^{m} \boldsymbol{\varepsilon}^{r} \cdot\left|\boldsymbol{\alpha}_{n-1+r}-\bar{y}^{(n-1+r)}(0)\right|+\boldsymbol{\varepsilon}\right)+ \\
& +C \boldsymbol{\varepsilon}^{n-q} e^{-\gamma \frac{t}{\varepsilon}}\left(\sum_{r=1}^{m-1} \boldsymbol{\varepsilon}^{r} \cdot\left|\boldsymbol{\alpha}_{n+r}-\bar{y}^{(n+r)}(0)\right|+\boldsymbol{\varepsilon}\right) \cdot\left|\sum_{k=1}^{m}(-1)^{2 n+m+k} \frac{\overline{\boldsymbol{\mu}}_{k}^{j}(t) \boldsymbol{\omega}_{m k}(t)}{\overline{\boldsymbol{\mu}}_{k}^{n+1}(0)}\right|, \\
& q=\overline{0, n+m-1} \text {. }
\end{aligned}
$$

where

$$
\begin{gathered}
a_{k}=\int_{0}^{1} \sum_{j=0}^{n+m-1} H_{j}^{(k)}(0, x) \boldsymbol{\eta}^{(j)}(x) d x, \\
a_{k i}=\int_{0}^{1} \sum_{j=0}^{n+m-1} H_{j}^{(k)}(0, x) \boldsymbol{\eta}_{i}^{(j)}(x) d x .
\end{gathered}
$$

Let $\Delta$ be a determinant of the system (39).

(C6) $\Delta \neq 0$. If the condition (C6) is valid, then the constants $B_{k}, \quad k=\overline{0, m-2}$ is defined singlevalued.

Theorem 3. Let assumptions (C1)-(C6) hold. Then the nonstandard unperturbed problem (21), (22) on the segment $0 \leq t \leq 1$ has an unique solution and is defined by the formula (37).

Let us denote by

$$
u(t, \boldsymbol{\varepsilon})=y(t, \boldsymbol{\varepsilon})-\bar{y}(t) \quad \Rightarrow \quad y(t, \boldsymbol{\varepsilon})=u(t, \boldsymbol{\varepsilon})+\bar{y}(t)
$$

Substituting (40) into the problem (1), (2), in view of the problem (21), (22), we get the following
The problem (41), (42) is of the same type as the problem (1), (2), by applying estimates (17), we get asymptotic estimations for the function $u(t, \varepsilon)$ as 
From the estimations (43), the following limiting equalities hold:

$$
\begin{aligned}
& \lim _{\varepsilon \rightarrow 0} u^{(q)}(t, \varepsilon)=0, \quad 0 \leq t \leq 1, \quad q=\overline{0, n} \\
& \lim _{\varepsilon \rightarrow 0} u^{(q)}(t, \varepsilon)=0, \quad 0<t \leq 1, \quad q=\overline{n+1, n+m-1} .
\end{aligned}
$$

Theorem 4. Let assumptions (C1)-(C6) hold. Then for the solution $y(t, \varepsilon)$ of the Cauchy problem (1), (2) the following limiting equalities hold:

$$
\begin{aligned}
& \lim _{\varepsilon \rightarrow 0} y^{(q)}(t, \varepsilon)=\bar{y}^{(q)}(t), \quad 0 \leq t \leq 1, \quad q=\overline{0, n,} \\
& \lim _{\varepsilon \rightarrow 0} y^{(q)}(t, \varepsilon)=\bar{y}^{(q)}(t), \quad 0<t \leq 1, \quad q=\overline{n+1, n+m-1,}
\end{aligned}
$$

where function $\bar{y}(t)$ is the solution of the nonstandard unperturbed problem (21), (22) and is defined by the formula (37).

\section{Conclusion}

The article is devoted to study the initial value problem for singularly perturbed the $n+m-$ th order linear integro-differential equation with a small parameters at the $m$-th derivatives. An explicit analytical formula of the solution of singularly perturbed initial value problem is obtained. The nonstandard degenerate initial value problem is constructed. The solution of the nonstandard degenerate initial value problem is obtained. The asymptotic convergence of solution of a singularly perturbed initial value problem to the solution of the nonstandard degenerate initial value problem is proved.

\section{Acknowledgement}

The authors were supported in parts by the MESRK grant AP05132587 "Boundary value problems for singularly perturbed differential equations with a continuous and piecewise constant argument" (2018-2020) of the Committee of Science, Ministry of Education and Science of the Republic of Kazakhstan.

\section{References}

1. Vishik I., Lyusternik L. A. "On the initial jump for non-linear differential equations containing a small parameter." Doklady Akademii Nauk SSSR 132, no. 6 (1960): 1242-1245.

2. Kassymov K. A. "On the asymptotics of the solution of the Cauchy problem with large initial conditions for nonlinear ordinary differential equations containing a small parameter." Uspehi Mat. Nauk 17, no. 5 (1962): 187-188.

3. Kassymov K. A. "The initial jump problem for non-linear systems of differential equations containing a small parameter." Doklady Akademii Nauk SSSR 179, no. 2. (1968): 275-278.

4. Kassymov K. A. "Asymptotics of solutions of Cauchy problem with an initial jump for nonlinear systems of integro-differential equations with a small parameter for the highest derivative." Izvestiya Akademii Nauk Kazakhskoi SSR no. 5 (1968): 69-72.

5. Kassymov K. A. "Asymptotics of the solution of a problem with initial jump for nonlinear systems of differential equations with a small parameter in the highest derivative." Doklady Akademii Nauk SSSR 189, no. 5 (1969): 941-944.

6. Kassymov K. A. "Asymptotics of the solutions of the problem with an initial jump for a system of differential equations of hyperbolic type with a small parameter at the derivative." Doklady Akademii Nauk SSSR 196, no. 2 (1971): 274-277.

7. Kassymov K. A. Linear singularly perturbed second-order differential equations. Almaty: 1981. $-112 \mathrm{p}$.

8. Kassymov K. A. "Boundary value problems with initial jumps for differential equations." Uspekhi Matematicheskikh Nauk 39, no. 4 (1984): 132-133.

9. Kassymov K. A., Abildayev E.A. "Asymptotic estimates for solutions of singularly perturbed boundary value problems with initial jumps for linear differential equations." Differential equations 28, no. 10 (1992): 1659-1669.

10. Kassymov K. A. Singularly perturbed boundary value problems with initial jumps. Almaty: Sanat, 1997. - 176 p.

11. Kassymov K.A., Nurgabyl D.N. "Asymptotic behavior of solutions of linear singularly perturbed general separated boundaryvalue problems with initial jump." Ukrainian Mathematical Journal 55, no 11 (2003): 1777-1792.

12. Kassymov K.A., Nurgabyl D.N. "Asymptotic estimates of solution of a singularly perturbed boundary value problem with an initial jump for linear differential equations." Differential Equations 40, no 5 (2004): 641-651. 
13. Kassymov K.A., Dauylbaev M.K. "Estimation of solutions of the Cauchy problem with initial jump of any order for linear singularly perturbed differential equations." Differential equations 35, no 6 (1999): 822-830.

14. Dauylbaev M.K. "The asymptotic behavior of solutions to singularly perturbed nonlinear integro-differential equations." Siberian Mathematical Journal 41, no. 1 (2000): 49-60.

15. Kassymov K.A., Dauylbaev M.K. "Singularly perturbed linear integro-differential equations with initial jumps of any order." Izvestiya VUZov. Matematika 494, no. 7 (2003): 70-75.

16. Dauylbaev M.K., Mirzakulova A.E. "Asymptotic behavior of solutions of singular integro-differential equations." Journal of Discontinuity, Nonlinearity, and Complexity 5, no. 2 (2016): 147-154.
17. Dauylbaev M.K., Mirzakulova A.E. "Boundary-value problems with initial jumps for singularly perturbed integrodifferential equations." Journal of Mathematical Sciences 222, no. 3 (2017): 214-225.

18. Dauylbaev M.K., Mirzakulova A.E., Ergaliev M.G. "Asymptotic behavior of solutions of the Cauchy problem for singularly perturbed differential equations with impulse action." Bulletin KazNPU named after Abay 49, no. 1 (2015): 24-29.

19. Nurgabyl D.N. "Asymptotic estimates for the solution of the initial value problem for linear differential equations with a small parameter for the highest derivatives." Bulletin ZhSU named after Zhansugurov no. 2 (2012): 4-8. 